\title{
The effect of seasonality on burn incidence, severity and outcome in Central Malawi
}

\author{
Anna F. Tyson ${ }^{a, c}$, Jared Gallaher ${ }^{a, b}$, Stephen Mjuweni ${ }^{a}$, Bruce A. Cairns ${ }^{b}$, \\ Anthony G. Charles ${ }^{a, b, *}$ \\ ${ }^{a}$ Department of Surgery, Kamuzu Central Hospital, Lilongwe, Malawi \\ ${ }^{\mathrm{b}}$ Department of Surgery, University of North Carolina at Chapel Hill, United States \\ ${ }^{\mathrm{c}}$ Carolinas Medical Center, Charlotte, NC, United States
}

A R T I C L E I N F O

Article history:

Received 4 October 2016

Received in revised form

30 January 2017

Accepted 31 January 2017

Keywords:

Burns

Burn

Seasonality of injury

Burn seasonality

Sub Saharan Africa

Malawi

\begin{abstract}
A B S T R A C T
Introduction: In much of the world, burns are more common in cold months. However, few studies have described the seasonality of burns in sub-Saharan Africa. This study examines the effect of seasonality on the incidence and outcome of burns in central Malawi.

Methods: A retrospective analysis was performed at Kamuzu Central Hospital and included all patients admitted from May 2011 to August 2014. Demographic data, burn mechanism, total body surface area (\%TBSA), and mortality were analyzed. Seasons were categorized as Rainy (December-February), Lush (March-May), Cold (June-August) and Hot (September-November). A negative binomial regression was used to assess the effect of seasonality on burn incidence. This was performed using both the raw and deseasonalized data in order to evaluate for trends not attributable to random fluctuation.

Results: A total of 905 patients were included. Flame (38\%) and Scald (59\%) burns were the most common mechanism. More burns occurred during the cold season ( $41 \%$ vs $19-20 \%$ in the other seasons). Overall mortality was $19 \%$. Only the cold season had a statistically significant increase in burn. The incidence rate ratios (IRR) for the hot, lush, and cold seasons were 0.94 (CI 0.6-1.32), 1.02 (CI 0.72-1.45) and 1.6 (CI 1.17-2.19), respectively, when compared to the rainy season. Burn severity and mortality did not differ between seasons.

Conclusion: The results of this study demonstrate the year-round phenomenon of burns treated at our institution, and highlights the slight predominance of burns during the cold season. These data can be used to guide prevention strategies, with special attention to the implications of the increased burn incidence during the cold season. Though burn severity and mortality remain relatively unchanged between seasons, recognizing the seasonal variability in incidence of burns is critical for resource allocation in this low-income setting.
\end{abstract}

\footnotetext{
* Corresponding author at: Department of Surgery, UNC School of Medicine, Gillings School of Global Public Health, University of North Carolina, 4008 Burnett Womack Building, CB 7228, United States. Fax: +1 9199660369.

E-mail address: anthchar@med.unc.edu (A.G. Charles).

http://dx.doi.org/10.1016/j.burns.2017.01.037
} 


\section{Introduction}

Injury is a leading cause of death worldwide and burn is disproportionally common in low and middle-income countries (LMICs), where 95\% of burns occur [1]. An estimated 300,000 deaths occur yearly from fires; and fire-related injuries are the 6 th leading cause of death in children aged 5-14 years old [2]. In addition, millions more suffer life-long disability and disfigurement from burns. These injuries are often attributable to cooking conditions and methods, poor access to safe energy source, crowded living conditions, and a lack of knowledge of potential risks [3]. Ultimately, burn is a disease of poverty.

Seasonality describes the cyclical fluctuation in time-series data. Describing seasonal patterns, as well as understanding the deviations from these patterns, is useful for predicting trends and planning resource allocation for services that may vary throughout the year. In most geographic regions with four distinct seasons, burns are more common in colder months, as people are more likely to use fires for both cooking and heating [4].

Despite the potential importance of seasonality in burn, available data on the seasonality of burn in sub-Saharan Africa are scarce. Among the many uses of injury surveillance programs is the potential to alert health authorities and communities to emerging injury trends, guide resource allocation, and facilitate development of prevention measures. This is particularly important in resource-poor settings, where limited resources must be distributed carefully.

Given the dearth of epidemiologic studies assessing seasonality in burn patterns in sub-Saharan Africa, the primary objective of this study is to evaluate seasonality in burn hospitalization and identify any temporal relationships in burn and outcome using data from a burn registry database in central Malawi.

\section{Methods}

\subsection{Setting}

This study is a retrospective analysis of prospectively collected burn registry data from patients admitted to the Kamuzu Central Hospital (KCH) burn unit from May 2011 to August 2014. $\mathrm{KCH}$ is an 800-bed tertiary care hospital in the capital city of Lilongwe, which serves as a referral center for approximately 5 million people in the Central Region of Malawi $[5,6]$. The burn unit at KCH was established in 2011 in collaboration with the University of North Carolina, Department of General Surgery. Pediatric and adult patients are admitted to the same unit, which consists of 31 beds and is staffed by 6 full-time and specially trained nurses and 2 clinical officers. There are no full-time plastic surgeons present at $\mathrm{KCH}$, although international plastic surgeons occasionally visit for short missions. Although there are general surgeons and general surgery residents engaged full time at the hospital, the majority of the clinical decision making and operative procedures performed in the burn unit are performed by 2 clinical officers who have received training in burn excision and skin grafting with consultant general surgeon oversight.

An average of 23 patients was admitted to the burn unit every month during the study period. A burn registry was established to collect patient demographics, clinical characteristics, and outcome. Specifically, variables utilized in this study include age, gender, season of injury, mechanism of injury, time to presentation to hospital, total body surface area (TBSA) burned, length of hospital stay, and survival. Once admitted to the burn unit, all patients are treated in a similar manner based on burn and associated medical issues.

\subsection{Seasonality}

Seasons were grouped into four 3-month periods: the rainy season (December-February), the lush, green season (MarchMay), the cold, dry season (June-August), and the hot, dry season (September-November). Average temperature fluctuates throughout the year from an average of $23.3^{\circ} \mathrm{C}$ during the hot season (range $17-29^{\circ} \mathrm{C}$ ) to an average of $18.3^{\circ} \mathrm{C}$ during the cold season (range $7-23^{\circ} \mathrm{C}$ ). The seasons in Malawi are generally predictable, although the lush, green season can vary in length, temperature, and amount of rainfall.

\subsection{Establishing seasonality and deseasonalizing the data}

For data influenced by seasonal trends, deseasonalizing the data serves to smooth the seasonal variations in order to look for irregularities in the pattern and allow for forecasting. This was performed by calculating burn incidence for each month during the study period and calculating a seasonal index using a three-mean centered average. The number of burns each month was then divided by the adjusted seasonal index in order to calculate the deseasonalized number of burns.

\subsection{Statistical analysis}

The initial cohort was described using means and percentages and bivariate analysis was used to compare patient characteristics, hospital course, and outcome between seasons. Pearson's Chi-square test was used to compare categorical variables. T-tests were used to describe normally distributed continuous variables and $\mathrm{K}$-sample equality-of-means test was used for highly skewed continuous variables. Negative binomial regression modeling was performed to compare the incidence of burn between seasons. Both the crude and deseasonalized data were included in the negative binomial regression in order to determine whether the observed differences were due to seasonality or random fluctuation. The results were described using incidence rate ratios (IRRdefined as the ratio between the observed and expected numbers of burn in each season) and $95 \%$ confidential intervals (CI).

For all calculations, a p-value of less than 0.05 was considered statistically significant. All statistical analysis was performed using Stata/SE 13 (StataCorp LP, College Station, TX). The University of North Carolina Institutional Review Board and the Malawi National Health Sciences Review Committee approved this study. 


\section{Results}

A total of 905 patients were admitted to the burn unit during the study period. Of these, roughly half were male and almost $80 \%$ were children less than 15 years old. Scald burns were the most common mechanism overall, accounting for almost $60 \%$ of all injuries. Approximately one-third of all burns were less than $10 \%$ TBSA and approximately $80 \%$ were less than $25 \%$ TBSA. Overall mortality was approximately 19\% (Table 1).

Characteristics of burns differed between children and adults (Table 1 ). In the pediatric population ( $<15$ years old), burns occurred almost evenly between males and females. Scald burns were the most common mechanism (68\%) followed by flame burns (31\%). In the adult population ( $\geq 15$ years old), two-thirds of burn victims were male and flame burns were more common than scald burns (70\% vs $25 \%$, respectively). The distribution of TBSA between the pediatric and adult populations was relatively similar, with most patients suffering less than 25\% TBSA burns; however, the adult population had a proportionally larger number of patients with severe burns greater than 50\% TBSA. Survival from burns was similar between the pediatric and adult populations.

Overall, $41 \%$ of burns occurred during the cold season, while the remainder of the injuries occurred evenly between the other seasons (Table 1). Scald burns were the most common mechanism of injury in all seasons except the cold season, when they were surpassed by flame burns (Table 2). Gender and age distribution did not differ significantly between seasons; likewise, there was no statistically significant difference in TBSA between seasons. In both the pediatric and adult age groups, burns were most common in the cold season (Table 1). Following binomial regression of both the crude and seasonally adjusted data in the adult and pediatric cohorts, the IRR revealed an increase in burn incidence during the cold season exceeding all the other seasons. Using the rainy season as the reference, there was a statistically significant increase in burn incidence in the cold season (Table 3). The IRR for the Hot, Lush and Cold seasons were 0.94 (95\% CI 0.6-1.32), 1.02 (95\% CI 0.72-1.45) and 1.6 (95\% CI 1.17-2.19), respectively. While the other seasons showed some seasonal variation in burn incidence, only the cold season demonstrated a statistically significant increase in burns (Fig. 1).

\section{Discussion}

This study establishes the seasonality in burn incidence in both the adult and pediatric burn patients in a sub-Saharan African setting. Our results demonstrate an increased burn incidence of approximately $60 \%$ in the cold season at our burn center as compared to the other seasons. However, despite variations in burn incidence, there is no substantial variability in survival for both adult and pediatric burn patients at this institution.

The pattern of seasonal variation in burn incidence in Malawi displays similarities with other countries in the region. A review of the literature from Nigeria indicates that seasonal variation is the most important influence on the prevalence of burns, with the winter months having the highest incidence. In

Table 1 - Characteristics of burn patients presenting to Kamuzu Central Hospital: May 2011-August 2014. a

\begin{tabular}{|c|c|c|c|c|}
\hline Characteristic & Total & Pediatric $^{b}$ & Adult $^{\mathrm{b}}$ & p-Value \\
\hline Total & 905 & $718(79 \%)$ & $187(21 \%)$ & \\
\hline Gender & & & & 0.001 \\
\hline Male & $55 \%$ & $52 \%$ & $66 \%$ & \\
\hline Female & $45 \%$ & $48 \%$ & $34 \%$ & \\
\hline Mechanism & & & & $<0.001$ \\
\hline Flame & $38 \%$ & $31 \%$ & $70 \%$ & \\
\hline Scald & $59 \%$ & $68 \%$ & $25 \%$ & \\
\hline Other & $2.4 \%$ & $1.6 \%$ & $5.4 \%$ & \\
\hline TBSA (\%) & $19(16)$ & $18(14)$ & $22(22)$ & 0.003 \\
\hline $0-10 \%$ & $36 \%$ & $35 \%$ & $39 \%$ & $<0.001$ \\
\hline $11-25 \%$ & $43 \%$ & $46 \%$ & $33 \%$ & \\
\hline $26-50 \%$ & $16 \%$ & $16 \%$ & $17 \%$ & \\
\hline$>50 \%$ & $4.8 \%$ & $3.3 \%$ & $11 \%$ & \\
\hline Season & & & & 0.90 \\
\hline Rainy (December-February) & $19 \%$ & $19 \%$ & $19 \%$ & \\
\hline Lush, green (March-May) & $20 \%$ & $20 \%$ & $19 \%$ & \\
\hline Cold, dry (June-August) & $41 \%$ & $41 \%$ & $41 \%$ & \\
\hline Hot, dry (September-November) & $20 \%$ & $19 \%$ & $21 \%$ & \\
\hline Survived to discharge & $81 \%$ & $82 \%$ & $77 \%$ & 0.1 \\
\hline
\end{tabular}

a Categorical variables: percentages may not add up to $100 \%$ due to missing data or rounding. Continuous variables: mean (SD).

b Pediatric $<15$ years old, adult $\geq 15$ years old. 
Table 2 - Patient characteristics based on seasonality of injury. ${ }^{\text {a }}$

\begin{tabular}{|c|c|c|c|c|c|c|}
\hline Characteristic & Total & Rainy & Lush, green & Cold, dry & Hot, dry & p-Value \\
\hline Total & 905 & $174(19 \%)$ & $182(20 \%)$ & 371 (41\%) & $178(20 \%)$ & \\
\hline Age $(\text { years) })^{b}$ & 9.9 (15) & $9.5(14)$ & $8.5(13)$ & $11(16)$ & $10(15)$ & 0.4 \\
\hline Pediatric $(<15$ yo) & $79 \%$ & $79 \%$ & $81 \%$ & $80 \%$ & $78 \%$ & 0.9 \\
\hline Adult ( $\geq 15$ yo) & $21 \%$ & $21 \%$ & $19 \%$ & $20 \%$ & $22 \%$ & \\
\hline Gender & & & & & & 0.5 \\
\hline Male & $55 \%$ & $55 \%$ & $59 \%$ & $56 \%$ & $51 \%$ & \\
\hline Female & $45 \%$ & $45 \%$ & $41 \%$ & $44 \%$ & $49 \%$ & \\
\hline Mechanism & & & & & & $<0.001$ \\
\hline Flame & $38 \%$ & $20 \%$ & $30 \%$ & $50 \%$ & $39 \%$ & \\
\hline Scald & $59 \%$ & $77 \%$ & $70 \%$ & $47 \%$ & $57 \%$ & \\
\hline Other & $2.4 \%$ & $3.6 \%$ & $0 \%$ & $2.5 \%$ & $3.5 \%$ & \\
\hline TBSA $(\%)^{\dagger}$ & $19(16)$ & $17(15)$ & $18(15)$ & $20(16)$ & $19(17)$ & 0.3 \\
\hline $0-10 \%$ & $36 \%$ & $36 \%$ & $34 \%$ & $34 \%$ & $41 \%$ & 0.2 \\
\hline $10-25 \%$ & $43 \%$ & $49 \%$ & $48 \%$ & $42 \%$ & $34 \%$ & \\
\hline $25-50 \%$ & $16 \%$ & $12 \%$ & $14 \%$ & $18 \%$ & $19 \%$ & \\
\hline$>50 \%$ & $4.8 \%$ & $2.7 \%$ & $3.9 \%$ & $5.9 \%$ & $5.1 \%$ & \\
\hline Time to presentation to hospital & & & & & & $<0.001$ \\
\hline $0-6 \mathrm{~h}$ & $27 \%$ & $24 \%$ & $24 \%$ & $27 \%$ & $33 \%$ & \\
\hline $7-12 \mathrm{~h}$ & $22 \%$ & $23 \%$ & $30 \%$ & $21 \%$ & $13 \%$ & \\
\hline $12-24 \mathrm{~h}$ & $15 \%$ & $22 \%$ & $17 \%$ & $14 \%$ & $7.8 \%$ & \\
\hline $24-48 h$ & $5.2 \%$ & $6.0 \%$ & $3.7 \%$ & $4.1 \%$ & $8.4 \%$ & \\
\hline$>48 \mathrm{~h}$ & $31 \%$ & $25 \%$ & $26 \%$ & $34 \%$ & $38 \%$ & \\
\hline Survived to discharge & $81 \%$ & $86 \%$ & $83 \%$ & $79 \%$ & $80 \%$ & 0.2 \\
\hline
\end{tabular}

a Seasons: Rainy (December-February), Lush/green (March-May), Cold/dry (June-August), Hot/dry (September-November).

${ }^{\mathrm{b}}$ Continuous variables: mean (SD).

\section{Table 3 - Seasonality of burns based on negative binomial regression. ${ }^{a}$}

Baseline no. burns per month $\operatorname{IRR}[95 \% \mathrm{CI}]$

\begin{tabular}{llcll} 
& & Lush & Cold & Hot \\
\cline { 4 - 5 } & 19 & $0.94[0.67,1.32]$ & $1.60[1.17,2.19]$ & $1.02[0.72,1.45]$ \\
Burns & 19 & $1.09[0.78,1.51]$ & $1.57[1.16,2.14]$ & $1.09[0.78,1.52]$ \\
Deseasonalized & 15 & $0.96[0.68,1.35]$ & $1.60[1.17,2.20]$ & $1.00[0.70,1.42]$ \\
Pediatric burns & 15 & $1.11[0.79,1.55]$ & $1.57[1.14,2.15]$ & $1.07[0.75,1.51]$ \\
Deseasonalized & 4 & $0.88[0.52,1.47]$ & $1.58[1.01,2.49]$ & $1.11[0.67,1.85]$ \\
Adult burns & 4 & $1.03[0.63,1.70]$ & $1.62[1.04,2.53]$ & $1.20[0.73,1.97]$ \\
Deseasonalized & 4
\end{tabular}

${ }^{a}$ Indicator variables were created for the seasons. The Rainy season (December-February) was used as the reference.

southern Africa, the use of open fires and paraffin lamps for heating and the use of hot water for bathing may contribute to the increased incidence of burns seen during the cold season [7-9]. As shown in our data, flame burns surpassed scald burns in the cold season, suggesting that the use of open fires for heating during the colder months may drive the increased burn incidence.

Despite the seasonal variation in burn incidence and mechanism, we did not observe any seasonal variations in burn severity as measured by TBSA or overall survival. Previous studies from this institution have demonstrated that the two main drivers of burn mortality in our environment are age and TBSA [10], both non-modifiable factors. As the age and
TBSA distribution do not vary significantly throughout the year, one might expect overall survival to remain relatively constant as well. However, the long-term morbidity from burns depends on the availability and adequacy of burn care resources and the fiscal constraints inherent in burn care delivery in resource poor countries [11,12].

Burn care is very resource intensive, using a supply of consumable dressings, medications, and operative costs for burn excision and grafting. At our institution, the estimated cost of burn care is 387 USD per day, or approximately 24 USD per patient per day [13]. In this resource poor environment, understanding of the seasonal variation of burn is paramount to ensure that operating theatres and burn units are equipped 


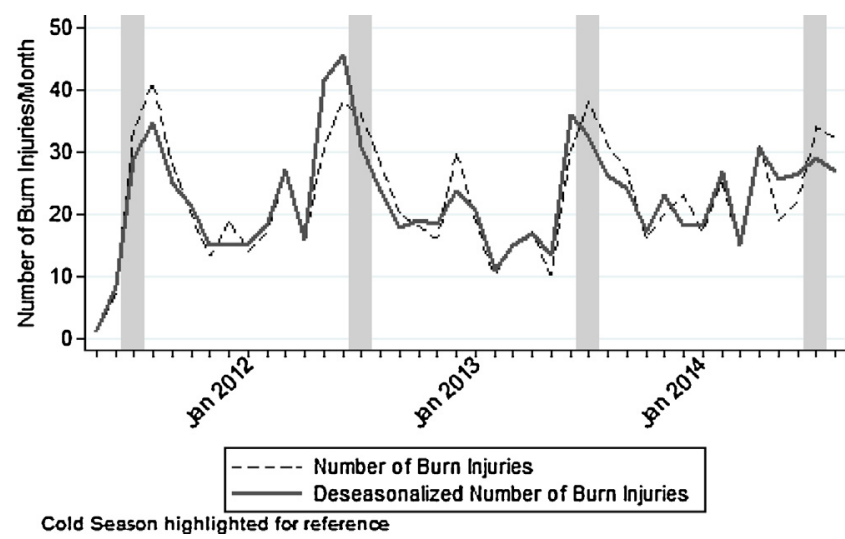

Fig. 1 - Frequency of burns (May 2011-August 2014).

to cope with the daily workload of burn care. This may mean setting aside money and non-perishable supplies throughout the year to be used during peak seasons.

Injury prevention strategies can also take into account seasonal variability in burns. At our center, pediatric patients account for approximately $80 \%$ of burn admissions. A lack of supervision of children during floor level cooking, bathing, or in the presence of open fires is common in our environment [14] and prevention efforts should be targeted to address these concerns. Mass campaigns at schools, churches, and other public gatherings should focus on improving burn awareness and may include education on fire enclosure, limiting the height of open flames in domestic environments, and improving the design of cook stoves, particularly with regard to stability and prevention of access by children [15].

The limitations of this study are those inherent to the retrospective methodology. The data analyzed is from a single center in the capital city of Malawi and hence may be subject to presentation bias. We cannot account for disparities in access, particularly in those residing in rural areas. Furthermore, the analysis was limited to hospitalized burn patients. We would expect the same surge in burn incidence to be reflected in the outpatient setting, but cannot confirm this based on the available data.

\section{Conclusion}

Burns in Malawi are a year-round phenomenon with a surge during the cold months. Management of burns presents a significant stress to the healthcare facilities at our institution. A thorough understanding of burn epidemiology, including seasonal variation, is critical not only for resource planning, but also for the implementation of targeted seasonal burn prevention initiatives.

R E F E R E N C E S

[1] WHO. World report on child injury prevention. Geneva: World Health Organization; 2008

[2] Mock C, Peck M, Peden M, Krug E. A WHO plan for burn prevention and care. Geneva: World Health Organization; 2008.

[3] Gevaart-Durkin A, Swart D, Chowdhury Z. A study of energyrelated injuries from hospital admissions among children and adults in South Africa. Burns 2014;40(6):1209-18.

[4] Hultman C, Tong W, Surrusco M, Roden K, Kiser M, Cairns B. To everything there is a season: impact of seasonal change on admissions, acuity of injury, length of stay, throughput, and charges at an accredited, regional burn center. Ann Plast Surg 2012;69(1):30-4.

[5] Samuel J, Campbell E, Mjuweni S, Muyco A, Cairns B, Charles A. The epidemiology, management, outcomes, and areas for improvement of burn care in Central Malawi: an observational study. J Int Med Res 2011;39(3):873-9.

[6] Kiser MM, Beijer G, Mjuweni S, Muyco AP, Cairns BA, Charles A. Photographic assessment of burn wounds: a simple strategy in a resource-poor setting. Burns 2013;39(1):155-61.

[7] Kalayi G. Epidemiology of burn injury: experience in the Savannah region of Northern Nigeria. Trop Doct 2001; 31:

209-11.

[8] Mobogunje O, Khwaje M, Lawrie J. Childhood burns in Zaria, Nigeria. Burns 1987;13:298.

[9] Niekerk AV, Rode H, Laflamme L. Incidence and patterns of childhood burn injuries in the Western Cape, South Africa. Burns 2004;30(4):341-7.

[10] Tyson AF, Boschini LP, Kiser MM, et al. Survival after burn in a sub-Saharan burn unit: challenges and opportunities. Burns 2013;39(8):1619-25.

[11] Greenwood J. Burn injury and explosions: an Australian perspective. Eplasty 2009;16(9):e40.

[12] Albertyn R, Numanoglu A, Rode H. Pediatric burn care in subSaharan Africa. Afr J Trauma 2014;3(2):61-7.

[13] Gallaher JR, Mjuweni S, Cairns BA, Charles AG. Burn care delivery in a sub-Saharan African unit: a cost analysis study. Int J Surg 2015;19:116-20.

[14] Bane M, Kaima R, Mapala S, Cairns B, Charles A. Qualitative evaluation of paediatric burn injury in Malawi: assessing opportunities for injury prevention. Trop Doct 2016;46(3):1657.

[15] WHO. Burns. [Accessed 14 April 2016]. 